\title{
The Effect of Oxygen on Freeze-dried Escherichia coli
}

\author{
By M. B. LION AND E. D. BERGMANN \\ The Israel Institute for Biological Research, Ness-Ziona, and Department \\ of Organic Chemistry, Hebrew University, Jerusalem, Israel
}

(Received 27 July 1960)

\begin{abstract}
SUMMARY
When Escherichia coli organisms were suspended in distilled water and freezedried the maximum loss of viability did not occur during the drying process proper, but during the time of contact of the dried organisms with air between the primary and the secondary drying periods. By substituting other gases for air at this stage, it was proven that oxygen was the active agent involved. The dried organisms which were exposed to different pressures of air and oxygen at different temperatures proved to be extremely sensitive to traces of oxygen, even at very low temperatures. The implications of this oxygen effect in connexion with existing freezedrying procedures, as well as some preliminary kinetic experiments concerning the shape of the survival curve, are discussed.
\end{abstract}

\section{INTRODUCTION}

High viabilities of bacterial suspensions after freeze-drying can usually be obtained only when the organisms are suspended in quite complex media during lyophilization. A survey of the literature (see, for example, Harris, 1954) shows that the discovery of these media has usually beeen a 'hit and miss' affair, and only in exceptional cases was an attempt made to rationalize the success of a certain medium (Fry \& Greaves, 1951; Naylor \& Smith, 1946). The lethal effect of freezing and drying on bacteria is indeed only very poorly understood, if at all. Studies of the nature of the damage caused by freezing different micro-organisms (Mazur, 1959; this article gives references to most of the current work) have failed to prove that freezing causes the principal or even any important damage during the process of lyophilization. Organisms which withstand freezing well may be killed by lyophilization when not protected by a suitable medium. In addition to freezing, there appeared therefore to exist one or more lethal stages in the drying process itself. An attempt to determine the viability of the bacteria, not only after the completion of the freeze-drying process, but also during its different stages, has apparently been made only once (Fry \& Greaves, 1951) and then, as will be shown further below, not under the most suitable conditions.

Most of the work dealing with lyophilization so far has been concerned with finding a medium in which the highest viability of the freeze-dried bacteria could be obtained. Under these circumstances it would be difficult to detect a phase of maximum killing, even if it existed, since when the overall viability of the freezedried bacteria remained comparatively high during the whole process, the statistical fluctuations in the viability determinations would be large enough to obscure a 
decrease in the viability during any one of the stages of freeze-drying. If one carries out the lyophilization of bacteria, which are resistant to freezing, under conditions under which high mortality is to be expected in the drying process, it should be possible to find out whether the mortality in this process is gradual, or whether it is confined to a single defined stage of the drying. As it has long been known (Fry, 1954; and confirmed by us) that freeze-drying of Escherichia coli in distilled water yields very poor results, we used this method to get high mortality in the lyophilization process. Moreover, freeze-drying from distilled water would leave the dried bacteria free from the components of the medium, and the results would be likely to be more generally valid. As the test organism has to be washed with, and suspended in, distilled water, the bacterium should be resistant to osmotic shock; E. coli has this property.

\section{METHODS}

Organism. A locally isolated strain of Escherichia coli, E/65, was used, unless otherwise indicated.

Culture methods. Organisms grown on freshly prepared nutrient agar slopes for $24 \mathrm{hr}$. at $37^{\circ}$, were washed off with, and re-suspended in, sterile nutrient broth to a concentration of approximately $10^{7}$ organisms $/ \mathrm{ml}$. This suspension was used to inoculate Roux bottles containing Difco nutrient broth solidified with about $2.5 \%$ (w/v) agar. After incubation for $20 \mathrm{hr}$. at $37^{\circ}$, the organisms were washed off into distilled water, sedimented at $20,000 \mathrm{~g}$, washed well with distilled water and then re-suspended in the same medium. This procedure was repeated. Finally, the organisms were resuspended in distilled water to a concentration of $1.5-2.0 \times$ $10^{11}$ organisms $/ \mathrm{ml}$. This suspension, from a sample of which a viable count was made, was then used for the lyophilization experiments.

Viable counts. Counts were performed by diluting a sample in gelatin buffer $(0.5 \%, w / v$, gelatine in $\mathrm{m} / 15$ phosphate buffer, $\mathrm{pH} \mathrm{6.8)}$. Portions $(0.5 \mathrm{ml}$.) of the appropriate dilutions were mixed with melted nutrient agar at $44^{\circ}$ in Petri dishes. At least three dishes were used at each dilution. After the agar had solidified, the dishes were inverted and opened on wire screens in a forced-air incubator for $15 \mathrm{~min}$. This procedure completely prevented spreading of colonies on the agar surface as well as between the agar and the bottom of the dish. The dishes were then closed and incubated for $48 \mathrm{hr}$. at $37^{\circ}$, and the colonies counted. Dilutions were selected so as to obtain between 50 and 500 colonies/dish.

\section{Lyophilization}

Apparatus. The apparatus used was of the simple manifold type. The ampoules were connected through high-vacuum rubber tubing to the manifold, the diameter of which was large enough to offer only negligible resistance to vapour flow. The condenser consisted of a stainless steel Dewar-type flask and was cooled by a solid $\mathrm{CO}_{2}+$ ethanol mixture. By a single stage rotary oil pump, a pressure of less than $10 \mu \mathrm{Hg}$ was produced; the pump was protected from water vapour by a phosphorus pentoxide trap. The pressure in the system was measured by a Pirani gauge connected to the system through a water vapour trap. Air which was bled into the system after lyophilization, was dried by passing it through sulphuric acid, 
soda lime and through a small-bore long $U$-tube immersed in a solid $\mathrm{CO}_{2}+$ ethanol mixture.

The secondary drying system, which was used in part of our experiments, consisted of a long large-bore horizontal cylinder which contained phosphorus pentoxide on stainless steel trays. Ampoules were connected to this system through manifolds. This system was evacuated by a double stage rotary oil pump to a pressure less than $0.5 \mu \mathrm{Hg}$.

Lyophilization procedure. The bacterial suspensions were distributed in $1 \mathrm{ml}$. amounts into (neutral) glass ampoules (outside diameter $15 \mathrm{~mm}$.; length $50 \mathrm{~mm}$.). The contents of the ampoules were quick-frozen in a solid $\mathrm{CO}_{2}+$ ethanol mixture and stored overnight at a temperature not exceeding $-70^{\circ}$. This storage did not affect the viability of the suspensions. The ampoules to be lyophilized were connected to the manifold while still immersed in the freezing mixture until a vacuum of at least $50 \mu \mathrm{Hg}$ was reached in the drying system. When this pressure had been obtained, the freezing mixture was removed and drying continued, the heat of sublimation being supplied by the surrounding atmosphere. Primary drying, as judged by the disappearance of moisture from the outside of the ampoule, was completed after 20-30 min. By that time the contents of the ampoule had been converted into a fluffy powder. The disappearance of moisture from the outside of the ampoule as criterion of dryness is only a very crude indication and depends among many other factors on the outside temperature and dew point. The drying was, therefore, continued for at least another 60-90 min. When secondary drying was required, dry air was bled into the system, then the ampoules were disconnected from the manifold, their necks drawn out in a small flame, and the ampoules were connected as soon as possible to the secondary drying system. After completion of the secondary drying the ampoules were sealed under vacuum.

For viable counts, the contents of the ampoules were reconstituted by adding gelatin + buffer immediately after opening the sealed ampoules, and the counts performed as soon as possible (never later than 20-30 min. after reconstitution, the material being kept meanwhile in the refrigerator).

\section{RESULTS}

To ascertain the point at which the bacteria were killed in the freeze-drying, viability curves were taken at every stage of the freeze-drying of a suspension of Escherichia coli in distilled water (freezing, primary drying, admitting dry air into the ampoules, drawing out and constricting the necks of the ampoules, secondary drying, sealing in vacuum). The counts were performed at intervals as short as technically feasible. The contents of three ampoules were reconstituted and their volume adjusted to that of the original sample. Table 1 represents the protocol of a typical experiment; the results are summarized in Fig. 1.

No significant change in viability occurred after freezing and storing in the frozen state, and only a small and gradual drop was found during the primary drying; at the end of that period $50 \%$ of the original population was still viable, although lyophilization was carried out in distilled water. On the other hand, a sudden drop in viability occurred between the end of the primary and the beginning of the secondary drying. During the secondary drying itself, there occurred only a small and gradual loss of viability. 
Table 1. Viability of Escherichia coli during lyophilization from distilled water

\begin{tabular}{|c|c|c|c|c|c|}
\hline \multirow[b]{2}{*}{ Manipulation } & \multicolumn{4}{|c|}{ Viable organisms/ml. } & \multirow{2}{*}{$\begin{array}{c}\text { Relative } \\
\text { viability } \\
(\%)\end{array}$} \\
\hline & Sample 1 & Sample 2 & Sample 3 & Average & \\
\hline Before freezing & $1.2 \times 10^{11}$ & $1.2 \times 10^{11}$ & $1.3 \times 10^{11}$ & $1.3 \times 10^{11}$ & 100 \\
\hline $\begin{array}{l}\text { After } 20 \mathrm{hr} \text {. at }-70^{\circ} \text { drying } \\
\text { starts }\end{array}$ & $1.2 \times 10^{11}$ & $1.1 \times 10^{11}$ & $1.2 \times 10^{11}$ & $1.2 \times 10^{11}$ & 92 \\
\hline- & $1.2 \times 10^{11}$ & $1.2 \times 10^{11}$ & $1.1 \times 10^{11}$ & $1.2 \times 10^{11}$ & . 91 \\
\hline 一 & $1.1 \times 10^{11}$ & $1.0 \times 10^{11}$ & $9.8 \times 10^{10}$ & $1.0 \times 10^{11}$ & 78 \\
\hline- & $9.8 \times 10^{10}$ & $9.4 \times 10^{10}$ & $1.1 \times 10^{11}$ & $9.8 \times 10^{10}$ & 77 \\
\hline - & $7 \cdot 9 \times 10^{10}$ & $9.0 \times 10^{10}$ & $8.7 \times 10^{10}$ & $8.5 \times 10^{10}$ & 67 \\
\hline End of drying; air admitted & $7 \cdot 8 \times 10^{10}$ & $8.1 \times 10^{10}$ & $5.0 \times 10^{10}$ & $6.3 \times 10^{10}$ & 50 \\
\hline Secondary drying starts & $9.7 \times 10^{9}$ & $6.4 \times 10^{9}$ & $6.4 \times 10^{9}$ & $7 \cdot 8 \times 10^{9}$ & $6 \cdot 1$ \\
\hline- & $5.8 \times 10^{9}$ & $4.2 \times 10^{9}$ & $\mathbf{3 . 8} \times 10^{9}$ & $4.6 \times 10^{9}$ & $\mathbf{8 \cdot 6}$ \\
\hline End of secondary drying & $5.8 \times 10^{9}$ & $6.1 \times 10^{\circ}$ & $3.7 \times 10^{9}$ & $5 \cdot 2 \times 10^{\circ}$ & $4 \cdot 1$ \\
\hline
\end{tabular}

The high mortality between primary and secondary drying may be ascribed to either of the following causes: (1) the change of pressure experienced by the organisms, when they were brought from vacuum to atmospheric pressure; (2) the effect of air per se. The rise in temperature of the dry powder during the constriction of the neck of the ampoule had no influence on the process.

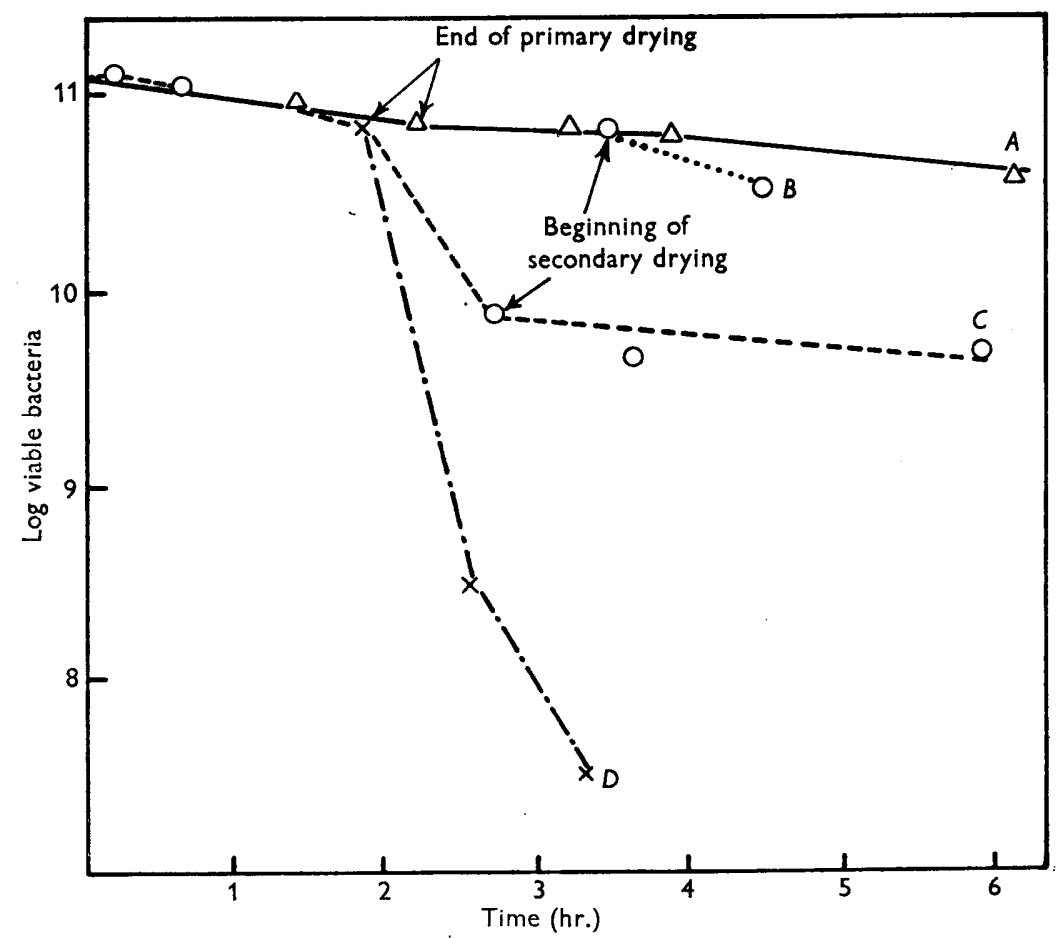

Fig. 1. Viability of Escherichia coli suspended in distilled water during the lyophilization procedure. The organisms were exposed to different atmospheres between the primary and the secondary drying: $A$, in vacuum; $B$ in hydrogen; $C$ in air; $D$ in oxygen. 
Organisms dried from distilled water were exposed to different gases at atmospheric pressure and at $28^{\circ}$. As controls, organisms were kept under vacuum for the same time and temperature. In view of the above results, the secondary drying was omitted. The results for hydrogen, air, oxygen, and vacuum for Escherichia coli E/65 are given in Fig. 1, and for oxygen, air and vacuum for $E$. coli B/r in Fig. 2. The results for these two organisms were similar, except that the $B / r$ strain appeared to be somewhat less sensitive. The viability counts for nitrogen were puzzling at first: the mortality was quite high as compared to hydrogen, although much lower than for air. Experiments showed that the purification of commercial nitrogen by

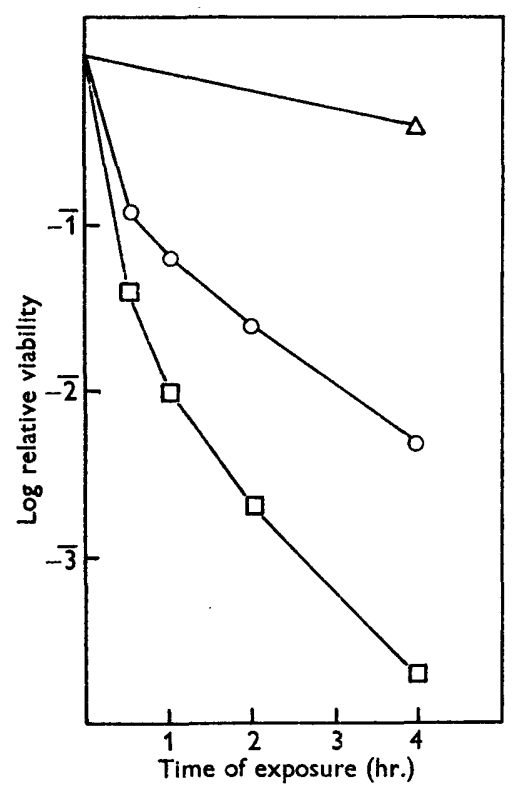

Fig. 2

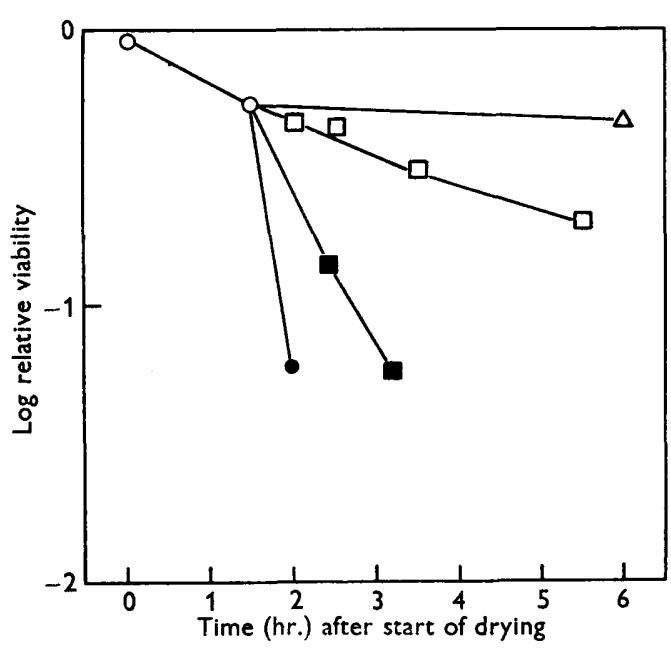

Fig. 3

Fig. 2. Exposure of Escherichia coli $B / \mathbf{r}$, dried from distilled water to vacuum, air or oxygen at $28^{\circ} . \triangle$, vacuum; $O$, air; $\square$, oxygen.

Fig. 3. Viability of Escherichia coli dried from distilled water and exposed to nitrogen 'purified' from oxygen by different methods. $\triangle$, vacuum; $O$, air; $\square$, nitrogen purified by Fieser's solution; $\mathbf{n}$, nitrogen purified by alkaline pyrogallol.

alkaline pyrogallol $(5 \%, w / v$, pyrogallol in a $50 \%, w / v$, aqueous solution of potassium hydroxide) was not efficient. When Fieser's solution (Fieser, 1924; $16 \%$, $\mathrm{w} / \mathrm{v}, \mathrm{Na}_{2} \mathrm{~S}_{2} \mathrm{O}_{4}, 4 \%$, w/v, sodium $\beta$-anthraquinone sulphonate in a $14 \%$, w/v, aqueous solution of sodium hydroxide) was used, better results were obtained. They are summarized in Fig. 3.

As the efficiency of the removal of oxygen from the nitrogen increased the viability of the dried suspensions exposed to nitrogen increased. On the other hand, even Fieser's solution did not seem to absorb all traces of oxygen under the conditions used by us. As will be shown presently, the suspensions dried from distilled water are extremely sensitive even to minute traces of oxygen. 
Effect of changes in the pressure of oxygen and in the temperature of exposure

Figure 4 shows that the viability of the dried suspension exposed to oxygen for $3 \mathrm{hr}$. at room temperature depended on the oxygen pressure. The extreme sensitivity of the suspensions to quite low oxygen pressures was pronounced; about $95 \%$ of these organisms were killed in $\mathbf{3} \mathrm{hr}$. by oxygen at a pressure as low as $10 \mathrm{~mm}$. Hg. (In these experiments part of the pressure range was covered by exposure to air, part by exposure to oxygen. Contrary to expectations, in the overlapping region of these two curves the results were not identical; it is not clear at present what caused the discrepancy.)

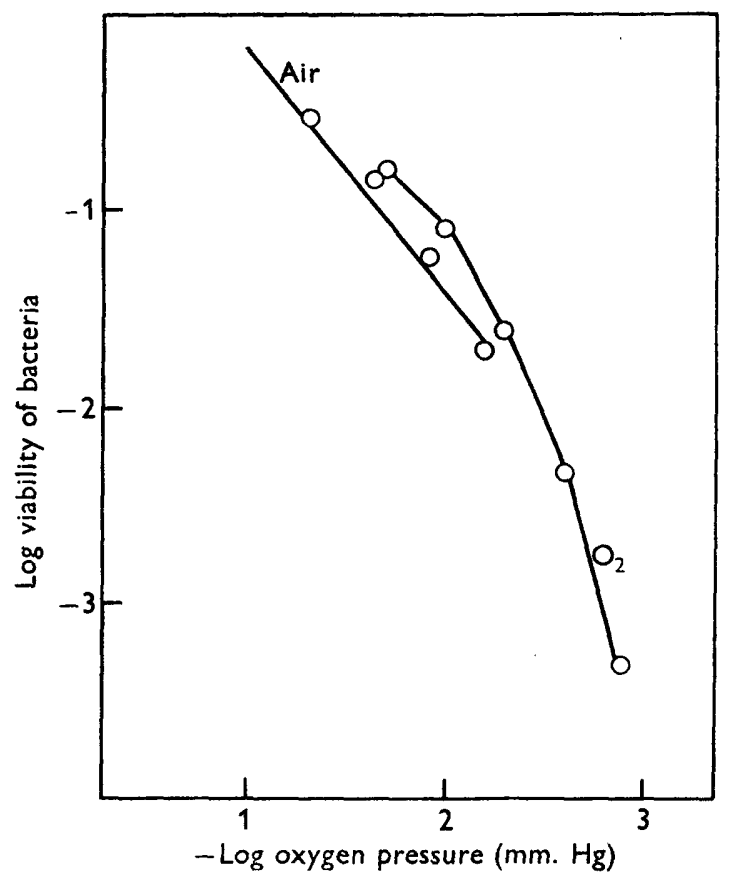

Fig. 4. Viability of Escherichia coli dried from distilled water and exposed to air and oxygen at different pressures for three hours at room temperature.

Figure 5 describes the viability of the dried suspensions when exposed to air at atmospheric pressure for $3 \mathrm{hr}$., but at different temperatures. Although the reaction between oxygen and the dried suspension was highly temperature-dependent, the mortality of the dried suspensions exposed to oxygen was easily measurable even at temperature as low as $-30^{\circ}$. When the exposure was prolonged beyond $3 \mathrm{hr}$., killing was evident at even lower temperatures.

\section{Shape of the survival curves}

As seen in Fig. 2, the plot of the logarithm of the survival in dried suspensions exposed to air or oxygen against time of exposure yielded a curve which is concave upwards. This may have been due to a consumption of oxygen and corresponding 


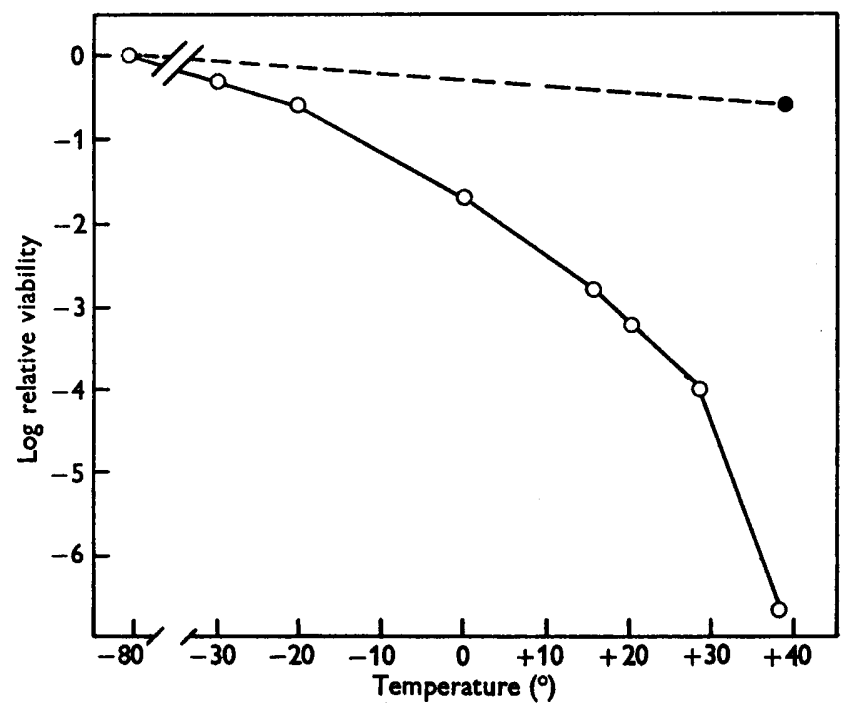

Fig. 5. Viability of Escherichia coli dried from distilled water and exposed to air at atmospheric pressure for three hours at different temperatures. 9 , control in vacuum; O, exposed to air.

fall in its pressure, which in turn would cause a slowing down of the reaction rate. That this is not the case is shown in Table 2, in which two conditions are compared: in one case the organisms were exposed to the normal volume of oxygen, while in the other a volume about twenty times as large was used. The results were not significantly different. In another experiment described in Table 3 different amounts of organisms were exposed to the same volume of oxygen. The results show that in

Table 2. Viability of Escherichia coli freeze-dried from distilled roater and exposed to different volumes of air at atmospheric pressure at $28^{\circ}$.

Original density of suspension $1.2 \times 10^{11} / \mathrm{ml}$. Each figure represents the average of three samples.

$\begin{array}{cccc}\begin{array}{c}\text { Volume } \\ \text { of air } \\ (\mathrm{ml} .)\end{array} & \overbrace{80} \begin{array}{c}120 \\ \text { Viability (\%) }\end{array} & 240 \\ 10 & 8.0 & 0.7 & 0.05 \\ 200 & 5.8 & 0.4 & 0.06\end{array}$

Table 3. Viability of Escherichia coli freeze-dried from distilled water at different concentrations of suspension, and exposed to air at atmospheric pressure at $28^{\circ}$.

Original

concentration of organisms

$1.5 \times 10^{11} / \mathrm{ml}$. $1.5 \times 10^{10} / \mathrm{ml}$.
Period of exposure to air (min.)

\begin{tabular}{|c|c|c|}
\hline 60 & $\begin{array}{c}120 \\
\text { Viability (\%) }\end{array}$ & 240 \\
\hline $1 \cdot 1$ & 0.3 & 0.08 \\
\hline $1 \cdot 4$ & 0.5 & 0.03 \\
\hline
\end{tabular}


this case the relative inactivation of the suspensions was independent of the original concentration of organisms. We see, therefore, that on the one hand the organisms were inactivated independently of each other, and on the other that the concentration of the inactivating agent was not rate-limiting under the conditions of our experiment. One would, therefore, expect an exponential survival curve. This point is discussed below.

\section{DISCUSSION}

The fact that oxygen is deleterious to lyophilized bacteria has been recognized for a long time (Rogers, 1914); for this reason freeze-dried substances are generally stored under vacuum or in inert gases. In some instances authors have tried to decrease the mortality in lyophilized material by adding anti-oxidants to the suspending medium (Naylor \& Smith, 1946); however, it is difficult to see how antioxidants are able to counteract the oxidation by traces of air in the dry state.

Experiments carried out to determine the effect of different gases on freeze-dried bacteria (Naylor \& Smith, 1946) demonstrated the lethal effect of air. This effect was a slow one and could be measured only after extended periods of storage; a $90 \%$ decrease in viable count was found for Serratia marcescens after storage in air for 48 days, as compared with an insignificant change when stored under vacuum. Interestingly enough, a decrease in viable count of $70-75 \%$ was found when these organisms were stored under tank nitrogen, or under nitrogen 'purified' (from oxygen) by alkaline pyrogallol. Similarly, unsatisfactory results with dry nitrogen were reported by Collier (1954). These results are not surprising in the light of the work described here.

Much more attention has usually been paid to the exclusion of moisture from the freeze-dried material than to the prevention of contact with air. Fry \& Greaves (1951) sealed their ampoules under vacuum, rather to exclude unknown amounts of moisture than to exclude traces of air. Swift (1937), Stillman (1941), and Morton \& Pulaski (1938) used dry air as storage atmosphere for their material, but this practice is no longer accepted (Fry, 1954). Meryman (1959) even suggested drying frozen material by passing dry air over it, instead of using the conventional vacuum. It seems, therefore, that the effect of oxygen on dried cells has been generally underestimated. In our work two facts have come to light. (1) It is possible to obtain a high survival in suspensions freeze-dried from distilled water, i.e. without any protective medium, when these suspensions are carefully protected from traces of oxygen. This is true at least in the case of Escherichia coli, which is resistant to hypotonic shock and to freezing in distilled water. (2) The failure reported by other workers in the lyophilization of suspensions from distilled water is due to the extreme sensitivity of the organisms to air. In the process of primary drying the neck of the ampoule should have a bore as large as possible. Since it is very difficult to seal such a wide neck under vacuum, the usual procedure is to let dry air into the system after the primary drying and to constrict the neck of the ampoule before the secondary drying. It is seen now that under these conditions drying of organisms from distilled water would not be successful. Substituting commercial nitrogen (even when 'purified' by conventional methods) for air between the primary and secondary drying, would not help very much, unless it is freed from oxygen by highly efficient reagents. Naylor's experiments (Naylor \& Smith, 1946), mentioned 
above, which demonstrated only a much smaller effect of air on freeze-dried suspensions, were performed on bacteria which were embedded in the drying medium devised by these workers. This would suggest that their medium contained a substance protecting the dried organisms from oxygen. This has indeed been proven to be the case (Lion \& Bergmann, unpublished).

Although careful exclusion of air prevented the death of most of the Escherichia coli organisms freeze-dried from distilled water, yet $50 \%$ were killed during the primary drying. This might be due to the traces of air diffusing through imperfect connexions of a vacuum system. Some air is also liberated during evacuation from the inner walls of the system, and furthermore, the small bubbles of air originally dissolved in water become trapped in the ice when the suspension is frozen and are liberated during freeze-drying when the ice evaporates. Although these traces of air may account fully for the loss of viability during the drying process, other causes of mortality are by no means excluded and, furthermore, the death of organisms during storage of freeze-dried material may occur even in the complete absence of oxygen. Complete absence of even traces of oxygen is extremely difficult to achieve, and these traces may have a long time in which to react with the organisms.

Some remarks about the shape of the survival curve depicted in Fig. 2 may be in order; its form is rather difficult to interpret kinetically. In our experiments oxygen was in excess. On the other hand, the relative inactivation rate seemed to be independent of the initial density of the suspension of organisms; thus no interaction between the organisms seemed to occur. Under these conditions, a first-order inactivation curve would be expected for a homogeneous population. As this was not the case, some heterogeneity must be present. The hypothesis that part of the population is genetically more resistant to oxygen is highly unlikely, since no mutant more resistant to freeze-drying (which nearly always includes such an oxygen effect) than the original population has ever been isolated. A metabolic or physiological heterogeneity cannot be excluded, although in our case it was not very probable, since the organisms were harvested long after the completion of the log phase and very carefully washed with distilled water before use. In general, our curve resembles very much the inactivation curve of poliomyelitis virus by formaldehyde. An analogous model to that proposed by Gard (1957) for this virus might be formulated with some justification. We believe, however, that more detailed kinetic studies should first be performed. The similarity between the inactivation curves of dried bacteria by oxygen and of virus by formaldehyde leads us to expect that the lethal effect of oxygen on unprotected freeze-dried bacteria may be utilized to prepare vaccines from killed bacteria. This method is now under investigation.

This paper forms part of a thesis submitted to the Hebrew University (by M.B.L.) in partial fulfilment of the requirements for the degree of Ph.D. 


\section{REFERENCES}

Collinz, L. H. (1954). In A Discussion on the Maintenance of Cultures by Freeze-Drying, p. 41. London: H.M. Stationery Office.

Freser, L. F. (1924). A new absorbant for oxygen in gas analysis. J. Amer. chem. Soc. 46, 2639.

Fny, R. M. (1954). The preservation of bacteria. In Biological Applications of Freezing and Drying, p. 215. Ed. R. J. C. Harris. New York: Academic Press Inc.

Fry, R. M. \& Gneaves, R. I. N. (1951). The survival of bacteria during and after drying. J. Hyg., Camb. 49, 220.

GARD, S. (1957). Chemical inactivation of viruses. In The Nature of Viruses; Ciba Symposium, p. 123.

Harris, R. J. C. (1954). Biological Applications of Freezing and Drying. New Yark: Academic Press Inc.

Mazun, P. (1959). Physical factors implicated in the death of microorganisms at subzero temperatures. Ann. N.Y. Acad. Sci. 85, 610.

Meryman, H. T. (1959). Sublimation freeze-drying without vacuum. Science, 130, 628.

Morton, M. E. \& Puxaski, E. J. (1938). The preservation of bacterial cultures. J. Bact. 35, 163.

NAyLOR, H. B. \& Smrth, P. A. (1946). Factors affecting the viability of Serratia marcescens during dehydration and storage. J. Bact. 52, 565.

Rogers, L. A. (1914). The preparation of dried cultures. J. infect. Dis. 14, 100.

Strillman, F. G. (1941). The preservation of Pneumococcus by freezing and drying. J. Bact. 42, 689.

SwIFT, H. F. (1937). A simple method for preserving bacterial cultures by freezing and drying. J. Bact. 33, 411. 